\title{
Optimization Analysis of Tennis Players' Physical Fitness Index Based on Data Mining and Mobile Computing
}

\author{
Shoudong Zhang ${ }^{1}$ and Huaqing Mao $\mathbb{D}^{2}$ \\ ${ }^{1}$ Sports Department, Jiangnan University, Wuxi, 214122 Jiangsu, China \\ ${ }^{2}$ School of Computer Engineering, Hubei University of Arts and Science, Xiangyang, 441053 Hubei, China \\ Correspondence should be addressed to Huaqing Mao; mr.maohuaqing@hbuas.edu.cn
}

Received 22 April 2021; Revised 10 May 2021; Accepted 18 May 2021; Published 2 June 2021

Academic Editor: Wenqing Wu

Copyright (C) 2021 Shoudong Zhang and Huaqing Mao. This is an open access article distributed under the Creative Commons Attribution License, which permits unrestricted use, distribution, and reproduction in any medium, provided the original work is properly cited.

\begin{abstract}
Tennis is a very explosive, continuous, and intense sport, including many continuous short-term explosive actions. It has the characteristics of short-term, high-intensity, high-density training, and it belongs to the category of purely competitive skills. In the competition, athletes must maintain good physical condition, physical fitness, and long-term endurance in order to demonstrate outstanding technical and tactical skills. Therefore, this paper proposes a mobile processor performance data mining framework MobilePerfMiner, which uses hardware counters and iteratively uses the XGBoost algorithm to build a performance model, ranks the importance of the microarchitecture events of the big data task, and reduces the performance big data dimension, so as to optimize the big data algorithm according to the performance characteristics described. Undoubtedly, the comprehensive monitoring of the sports training process is complex system engineering. The main monitoring includes three aspects: physical condition, technical and tactical skills, and intelligence. Sports technology is reflected in the ultimate load. According to the convenience and actual needs of the research, this article will discuss the methods of evaluating tennis training load and the actual technical and tactical parameter characteristics that can be obtained by studying the characteristics of tennis, namely, kinematics. Parameters for noncontact testing, the next step is to discuss the appropriateness and necessity of the load, as well as the technical and routine monitoring of tennis training ability. The final experimental results show that it can improve the physical energy of tennis players by more than $17 \%$.
\end{abstract}

\section{Introduction}

The rapid development of big data is profoundly changing people's production and life and changing the world. In recent years, with the increasing number of mobile devices and the increasingly powerful computing power of mobile processors, it has become more common to run big data tasks on mobile platforms. Running big data applications on mobile platforms helps developers quickly apply new big data algorithms to mobile platforms, enabling people to obtain big data services anytime and anywhere, improving people's production and life, and promoting industrial transformation [1].

Since Chinese tennis started late on the international stage, its relative level is low, and its growth rate is slow; one of the main reasons for this result is the lack of popularity of tennis. Many athletes are slow in contact with tennis, so they miss the fast-growing fitness time, which hinders the development of tennis in the later period. New tennis players are the hope and new strength of the development of tennis in our country. Slowly they only start with basic knowledge. There will be many top tennis players. Therefore, tennis training for young people is the top priority, and the country has begun to pay attention to it. This has helped to carry out in-depth youth tennis training, and won many competitions and opportunities, and provided young tennis players with more opportunities.

Yang established a volleyball fitness model based on data mining, used mathematical statistics to analyze the five main components of volleyball players, and obtained volleyball representative volleyball indicators based on the five fitness characteristics. Based on the adaptability of various indicators, the ratio of the quality factor and the characteristic value 
of the rotated index to the total characteristic value of the various factors can be used to obtain the weight coefficient of a healthy athlete representing the weight index of the physical index. Through the weighting factor, the physical fitness model of volleyball players based on data mining can be obtained. Using this model can effectively evaluate the athlete's training, competition, and health status. The factor analysis method is used to test the planned health evaluation model of volleyball player data mining, and the results show that the evaluation model is reliable. However, the experimental data is too few and not convincing enough [2]. In order to study and test the success factors of women's tennis matches, Yulin established bibliographic data methods, mathematical statistical methods, and decision tree prediction models to conduct statistical analysis on the data of 198 unemployed women from four continents. Research shows that the accuracy of the decision tree prediction standard model is $91.4 \%$, and the new decision tree model is feasible. The main factor that determines a woman's chance of winning a tennis match is the percentage of receiving and scoring. The result of $41 \%$ shows that the rate of receiving and scoring is the lifeline for women to play tennis; $41 \%$ of service scores and $61 \%$ of first service scores are lower than 28 non-Mandatory errors which have the highest win rate, $96.7 \%$. The conclusion is that technology sending and receiving is the link between current tennis technology and the key factors of the game result. Now, women playing tennis must pay attention to efficiency and reduce nonmandatory errors. The main ways are to improve the winning rate of women's tennis matches, provide appropriate countermeasures and suggestions to check the quantitative indicators of tennis match winners, and provide specific and effective implementation methods for tennis training. However, the data model he selected is not complete, and the data should be randomly selected for experiments [3]. Yang and Yan believe that diagnosing the scientific state of the tennis training process is the key link to improve the training level and competitive performance of tennis players. It is necessary to receive physical education through the reference index of the ability test of the university graduate school, train outstanding tennis players in the sports college, and carry out effective game monitoring. And method conclusions determine the physical condition and composition of excellent tennis players and other physiological indicators. The Graduate School of the School of Physical Education is aimed at understanding the physical functions of outstanding tennis players in the School of Physical Education, at providing scientific support for the tennis level and athletic performance of students across the country, at training their tennis enthusiasts, and at improving the overall improvement of ordinary tennis players. However, the amount of data is too large, and the data statistics process is very difficult [4].

The research goal of this work is to monitor mobile processor microarchitecture events when running big data tasks and use data mining methods to analyze event values and then characterize the performance characteristics of big data tasks on mobile processors. The final research goal of this work is to improve the performance of mobile processors from the perspective of characterizing the performance char- acteristics of big data tasks and propose a plan for improving the physical fitness of tennis players. The computing power of modern mobile processors is getting stronger and stronger, and more and more applications are deployed on mobile platforms. How to measure and understand the performance characteristics of applications on mobile platforms is the main content of this research. This work structured event features and merged events as features. Structured means that the original data obtained by monitoring needs to be cleaned and corresponds to the feature names one to one; the reason for using events as features is that we hope to find events that affect system performance, that is, a factor that affects system performance, so events are taken as characteristics of system performance; merging refers to merging the event values obtained from each monitoring and making full use of the event values of each monitoring. Finally, the relationship between microarchitecture events and performance is established, and the event characteristics are sorted, so that we can find events that affect system performance.

\section{Optimization Method of Tennis Player's Physical Fitness Index}

This article takes the core elements of the long-term training model of tennis players as the research object, namely, the "ten-year phenomenon," "specialized timing," and "window of training opportunity" [5]. It demonstrates its rationality from two levels of theory and practice and its impact on my country's tennis talents, the guiding significance of training [6].

2.1. Literature Data Method. During the research process, more than 200 related documents were reviewed using a bibliographic search engine, collected, localized, and classified, including related documents about their current status [7]: 39 articles about the training of domestic and foreign athletes, 56 articles about the LTAD model, 8 articles about the LTAD tennis model, 18 articles about the retention of sports at home and abroad, 23 articles about the "decade phenomenon," and about the 20 of the 26 articles of "Century Phenomenon" related to the topic of specialization time and 19 related studies on "windows of training opportunities" [8]. Since there are relatively few studies on the LTAD model in China, it was carried out in the early stage of this study. A lot of foreign language reading work combined with knowledge of sports coaching, teaching methods, sports psychology, sports anatomy, etc., summarized the literature and knowledge points related to this article, and created a knowledge system and preliminary information for this article [9].

2.2. Empirical Research Method. Taking elite athletes as the research object and as their talents develop, collect information and data consistent with this article [10]. For example, when we investigated the elucidation of the "ten-year phenomenon" in the training of tennis talents in my country, we collected some data, such as the age of participation of famous tennis players and the performance age of the National Games to support the "ten-year phenomenon" [11]. When we investigated the clarification of the "timing of specialization" for training tennis talents in our country, 
we checked a lot of historical data about talent training and found that the phenomenon of "early specialization" existed in the early stage. During the training process, while studying the inspiration of "Window of Opportunity" in cultivating excellent tennis talents in our country, we conducted retrospective interviews with coaches on various key characteristics of the training process of excellent tennis players and highlighted the sensitive time the paragraphs are relatively consistent, and a certain number of references have been found $[12,13]$.

2.3. Expert Interview Method. This research conducted a survey in the form of face-to-face interviews. First, the interviewed experts were introduced to the background, purpose, and significance of the interview, and then the experts were asked to answer and discuss the questions in the interview [14]. Time after time, summarize and clarify the main content of each question in the interview, use a tape recorder to record the interview process of the interviewed experts, and organize the data set [15]. The main content of the interview is based on the main part of the article: the basic elements of the long-term coaching model of tennis players, namely, "decade phenomenon," "special period," "window of training opportunities," three aspects, and years of athlete training and information experience and experts' interview $[16,17]$.

\subsection{Data Mining Process}

(1) Defining the problem according to the problems and needs of the enterprise: select the data mining target, through the precise positioning of the enterprise problem; select the data type and prediction model, so as to design a targeted marketing strategy; the main steps include business needs analysis, data relationship selection, business model association, and prediction model selection. Figure 1 shows a picture of a tennis player hitting the ball [18]

(2) Preparing the data [19]: the data set plays a decisive role in the mining model. Because the data format in the original data set is complex and diverse, there are often incorrect data, outliers, or dirty data, which cannot meet the data requirements of modeling [20]. Therefore, in order to improve the data quality, match the data requirements of the model and preprocess the data. The specific steps include data cleaning, data integration, data reduction, data transformation, and data dimensionality reduction, so as to extract structured data indicators related to the topic [21]. A follow-up analysis work provides effective data support

(3) Exploring data: exploring data is a key part of the data mining process. Data exploration is generally divided into classification and clustering [22]. The classification process is to train the data set by using the classification algorithm and then use the test data

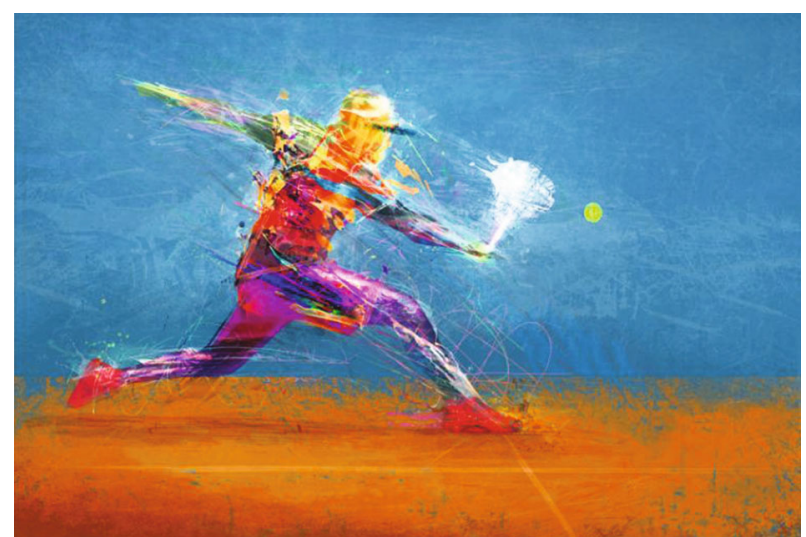

FIgURE 1: Tennis player hitting the ball (the picture comes from https://image.baidu.com/).

set to evaluate the accuracy of the selected classification rules. Clustering is to select a partition method to classify the data according to a certain standard and then test the accuracy of the method [23]

(4) The mining modeling (classification, clustering, association, and prediction): models are diverse, and analysis models are established according to specific needs, and the data content is researched and explored [24]. In addition, the model creation should match the data of the mining structure columns and link the data structure to the data set. And the data set that conforms to the mining structure will be matched to the database and become valuable data $[24,25]$

(5) Model verification and evaluation: the ultimate goal of the model is to have commercial value [26]. Before the model is put into practical application, the performance of the model should be tested and evaluated in many aspects, and the test results should be understood and analyzed in combination with the practical application, and the results should be discovered [27]. Valuable business models can be compared and analyzed on multiple similar models to determine which model best meets the data requirements of the problem, to determine the feasibility of the model [28].

\section{Correlation Experiment of Data Mining and Mobile Computing}

3.1. Introduction to XGBoost Algorithm. The XGBoost algorithm is a tree-based lifting algorithm. It is a new tree learning algorithm for processing sparse data based on the gradient boosting tree method (GBM or GBRT (gradient boosting machine or gradient boosted regression tree)), a theoretically reasonable weighted quantile calculation program The instance weight can be handled in the approximation tree learning. Use scaling and column sampling to improve the model's ability to fit data. 
For a given data set of $n$ samples and $m$ features, $D=(x$ $, y)$ :

$$
\hat{y}=\Phi\left(x_{i}\right)=\sum_{k=1}^{k} f\left(x_{i}\right)
$$

$q$ represents each tree structure and maps a sample to the corresponding page index. $T$ is the number of leaves in the tree. Each $f$ corresponds to an independent tree structure $q$ and leaf weight $w$. Unlike decision trees, each regression tree contains a continuous score on each leaf node, and $w$ represents $i$-th leaf score.

$$
\begin{gathered}
\wedge(\varphi)=\sum_{i} L\left(y_{i}, x_{i}\right)+\sum k\left(f_{k}\right), \\
\prod(f)=\sum_{i} l\left(\hat{y}^{t-1}+f_{t}\left(x_{i}\right)\right)+f_{k} .
\end{gathered}
$$

Among them, $L$ is a differentiable convex loss function, used to measure the distance between the predicted value $y$ and the true value. The second term of the formula is the penalty term for model complexity (such as the number of functions of the regression tree) [29]. The specific process of data mining is shown in Figure 2.

The accumulation rule is to help adjust the final learning rate to avoid overfitting. Instead, the model uses an accumulation operation for training.

$$
\Delta(\varphi)=\sum_{i} l\left(\hat{y}, y_{i}^{t-1}+f_{t}(x)\right)
$$

This means that we greedily accumulate $t$ according to formula (3) to improve our model the most, and the second-order approximation of the objective function can be quickly optimized.

$$
\Pi^{t}=\sum_{i=1}^{n}\left[l\left(y_{i}, \hat{y}\right)+g_{i} f(x)+\frac{1}{2} h_{i} f^{2}(x)\right]
$$

Among them,

$$
g_{i}=\partial(t-1) l\left(y_{i}, y^{t-1}\right)
$$

It is the first-order and second-order statistics of the loss function; after deleting the constant term, we get

$$
\lambda^{t}=\sum_{i=1}^{n}\left[g_{i} f\left(x_{i}\right)+\frac{1}{n} h_{i}+\gamma T\right]+\frac{1}{2} \lambda .
$$

The above formula can be used as a scoring function to measure the quality of the tree structure $q$. This score is similar to the purity score of the evaluation decision tree, except that it is derived for a broader objective function [30].

3.2. XGBoost Algorithm and Feature Selection. Performance counters provide information about the state of the processor and can be used to detect the execution and performance of

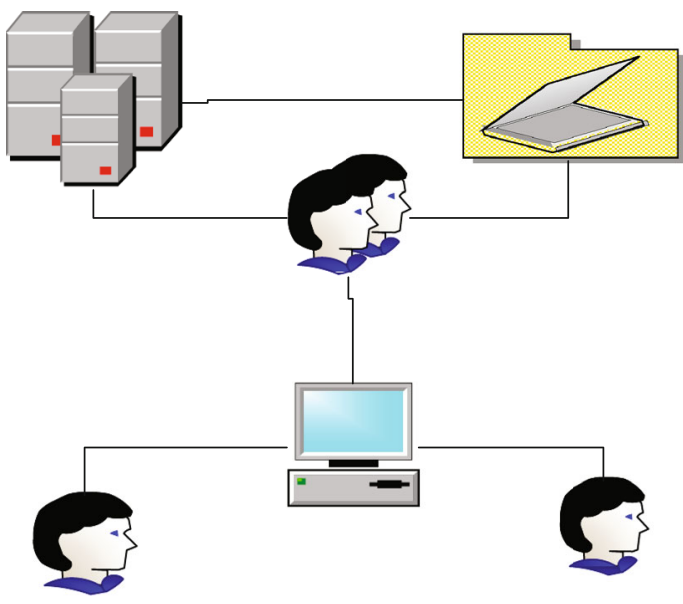

FIgUre 2: The specific process of data mining.

the workload. However, the link between the counter value and the observed performance is difficult to capture. On the one hand, the complexity of the processor makes it an arduous task to interpret the measured microarchitecture events and performance. On the other hand, the microarchitecture events supported by the processor are numerous and complicated. How to choose fewer microarchitecture events? And how to establish the connection between microarchitecture events and performance is the research goal of the MEE module.

$$
\operatorname{IPC}=\operatorname{perf}\left(e_{1}, e_{2}, \cdots, e_{n}\right)
$$

$e$ is a certain microarchitectural event and IPC is how many instructions are executed in each cycle, which is an important indicator of performance and $n$ is the number of microarchitectural events. Through the establishment of XGBoost algorithm model for events and performance, XGBoost is good at processing sparse data and high-dimensional data and characterizes the processor performance characteristics of running big data tasks on ARM processors. And use the feature importance obtained by the XGBoost modeling process to sort the events.

$$
\left.\prod_{l}^{2}(T)=\sum_{t=1}^{l-1} l_{t}^{2} I(v(t))=l\right)
$$

This importance metric can easily be extended to additive tree expansion, which simply averages the tree.

$$
\prod_{l}^{2}=\frac{1}{M} \sum_{m=1}^{M} T_{m}
$$

For each $J$ in the hidden layer or output layer, the net input and input of the calculation unit $J$ are relative to the previous layer $I$ :

$$
I_{j}=\sum_{i} w_{i j} O_{i}+\theta
$$


TABLE 1: Descriptive statistics of male athletes of different ages.

\begin{tabular}{lcccc}
\hline & Age & Number of cases & Average value & Standard deviation \\
\hline Seat body forward bending performance & $16-18$ years old & 39 & -12.938 & 7.5906 \\
Throwing a solid ball (2 kg) with both hands & $16-18$ years old & 30 & 11.3213 & 2.13373 \\
1 minute double-flying rope skipping results & $16-18$ years old & 35 & 73.50 & 35.675 \\
Fan running results & $16-18$ years old & 40 & 16.6015 & 1.05556 \\
800 m score & $16-18$ years old & 43 & 27.85 & 19.780 \\
\hline
\end{tabular}

Because the BP neural network has the advantages of nonlinear mapping ability, self-learning and adaptive ability, generalization ability, fault tolerance, etc., it has a wide range of applications in the fields of function approximation, pattern recognition, data prediction, etc. Each unit of the calculation output layer error selects the sigmoid function as the excitation function:

$$
\operatorname{Err}_{i}=O_{i}\left(1-w_{j}\right)\left(T-m_{i}\right)
$$

Aiming at the problems of BP neural network algorithms that are easy to fall into local extremes, slow convergence speed, and low optimization accuracy, there are many improvement methods at present. This paper uses the BP neural network model based on the improved PSO algorithm and the GA algorithm to predict mobile user behavior. The improved BP neural network model training has a faster convergence speed and a more accurate algorithm. The improved BP neural network algorithm is used to predict the behavior of mobile users, and the accuracy and stability are significantly improved.

\section{Optimization Analysis of Tennis Player's Physical Fitness Index}

4.1. Specific Physical Fitness of Young Tennis Players. Flexibility refers to the movement ability of human joints in different directions and the stretching ability of soft tissues such as muscles and ligaments. Flexibility is expressed through the range of joint motion, which is also reflected in the range of motion generated by a certain axis of motion.

Table 1 shows the descriptive statistics of various physical qualities of male athletes in different age groups. The table mainly lists the number of male athletes of each age group, the average value, and the standard deviation. The left and right hand solid balls improve with age. The 1-minute double-flying rope jumps better with age. The fan-shaped run and the 800-meter run also increase with age; the faster the running speed, the shorter the time. Under normal circumstances, specific physical fitness improves with age and conforms to the physiological laws of the human body, except for sitting forward bending.

It can be seen from Table 2 that in the intragroup comparison of the physical flexibility of male group A tennis players, the $P$ values in the same group are all greater than 0.05 , which indicates that the top eight male players in group A are compared with the non-top eight players. The difference in physical flexibility of famous athletes is not obvious.
TABle 2: Comparison of the difference between men's group competition performance and seat bending performance.

\begin{tabular}{lcccc}
\hline $\begin{array}{l}\text { Whether the top } \\
\text { eight }\end{array}$ & $\begin{array}{c}\text { Number of } \\
\text { cases }\end{array}$ & $\begin{array}{c}\text { Average } \\
\text { value }\end{array}$ & $\begin{array}{c}\text { Standard } \\
\text { deviation }\end{array}$ & $\begin{array}{c}t \\
\text { value }\end{array}$ \\
\hline Yes & 8 & -15.563 & 6.6586 & -1.100 \\
No & 32 & -12.261 & 7.7670 & n.s. \\
\hline
\end{tabular}

From Figure 3, the 16-18-year-old male athletes in Group A are among the top eight in the seat body who bend forward and throw a solid ball with both left and right hands, 1minute double-flying rope skipping, fan-shaped running, and 800-meter performance difference comparison table found. The top eight male athletes in the 16-18-year-old group have achieved a significant level in the $t$-statistics of each performance test of throwing a solid ball with both left and right hands, a 1-minute double flying skipping rope, and 800 meters. The significant probability value $P$ is all less than 0.05 , which means 16 . Whether the top eight male athletes in the 18-year-old group have a solid ball with their right hands, a 1-minute double-flying rope, and 800 meters are significantly different.

It can be seen from Figure 4 that, first of all, experts particularly emphasize the cultivation of athletes' will and quality in training. Long-term professional sports training must be boring, and it is a great test for athletes' physiology and psychology. Because young athletes are in the developmental stage, their minds are also showing relatively immature characteristics. Most athletes love to "play" and can persist with the support of hobbies at the beginning of training. As the training content deepens and the amount of training increases, the training process inevitably tends to be repetitive and boring. If there is not enough mental motivation and will quality, it is difficult to persist. This requires coaches and parents to need more encouragement and support in training and conveys to them the basic concept of "training for oneself," so that they can generate independent restraint, so that the effect of training can be improved.

As shown in Figure 5, the overall curve describes the growth process of the body in terms of height and weight. It includes the growth of various systems of the body, such as the muscles, bones, lungs, heart, ligaments, and tendons. The shape of the curve indicates the slow and steady development of body structure or figure between the ages of 5 and 12 . Simply put, this period is the best period for the development of basic motor skills. Neural curves describe the development of the brain and nervous system. At about 7 years old, $95 \%$ of 


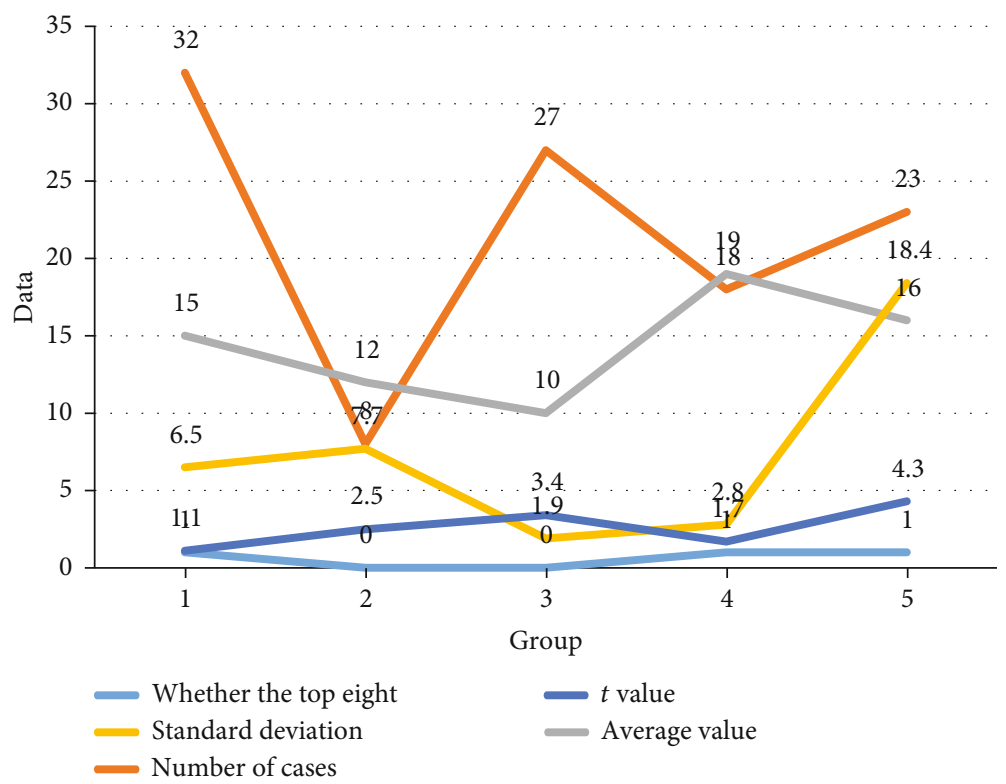

Figure 3: Comparison of the difference between male A group competition results and specific physical fitness results.

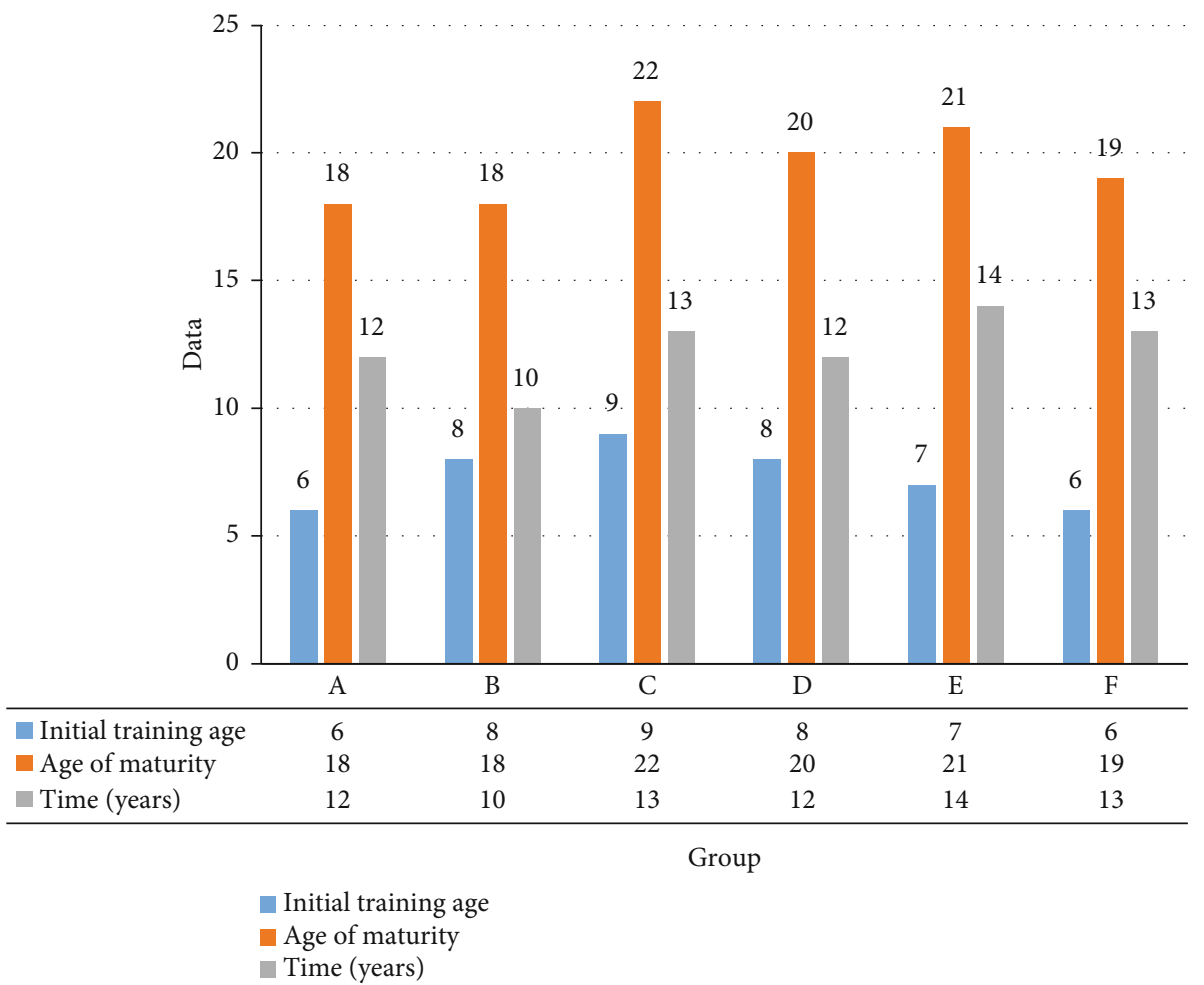

FIgURE 4: Table of the age of excellent tennis players at the beginning of training and the age of outstanding results.

the central nervous system has been developed. The shape of the nerve curve indicates that children should be trained early on motor skills such as agility, balance, coordination, and speed. The reproductive curve shows the growth pattern of the first and second sexual characteristics. The growth of reproductive tissues is slow, there is an incubation period in childhood, and it is extremely rapid before the growth spurt in adolescence. This curve shows that hormone maturation has a significant increase in physical development and ability improvement.

4.2. Feature Extraction of Tennis Microarchitecture Events. By observing the importance ranking of the microarchitecture events of Spark-bench benchmarks, we have obtained the characteristics of four types of programs, which are common and individual characteristics, memory characteristics, 


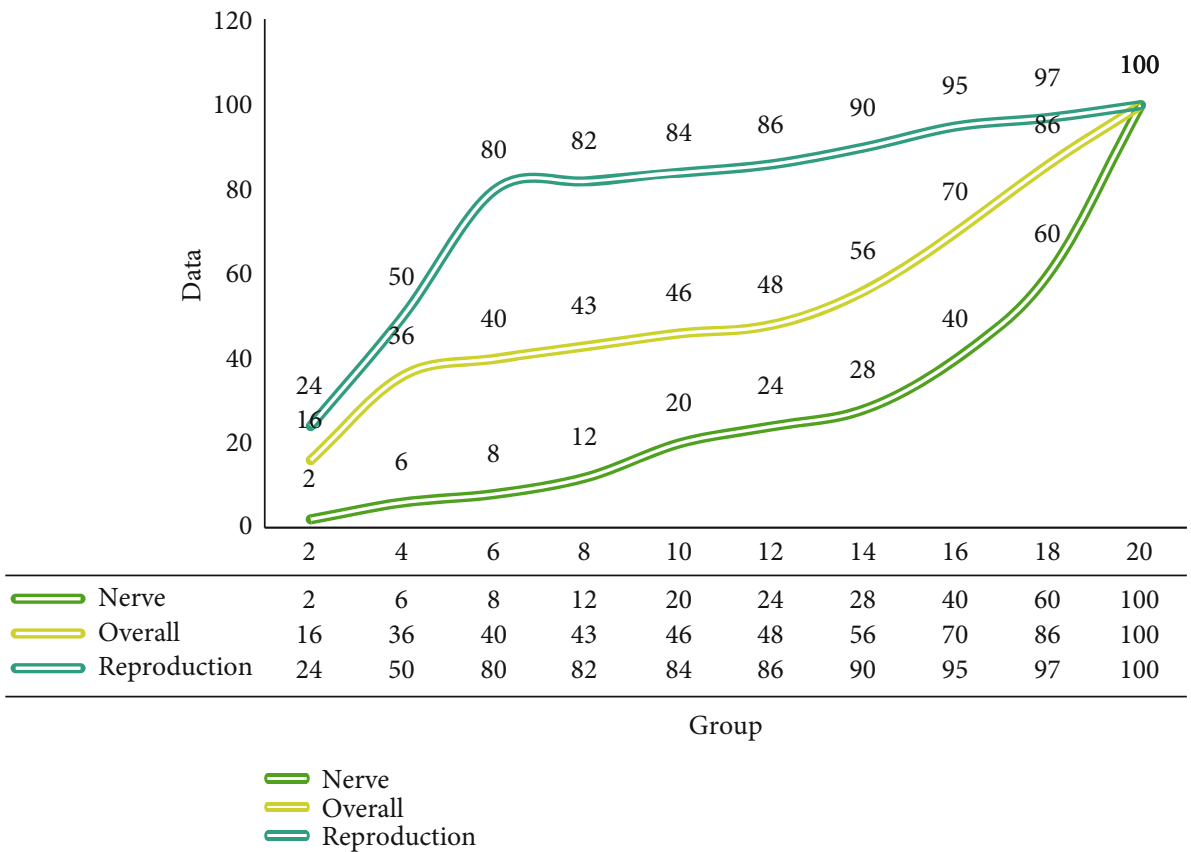

Figure 5: The curve of the human body's overall, neurological, and reproductive development trajectory.

TABLE 3: Event name and description.

\begin{tabular}{lcc}
\hline Abbreviation & Event name & Description \\
\hline BIR & BR_IMMED_RETIRED & Immediate branch instruction \\
BMP & BR_MIS_PRED & Prediction errors or unpredictable branch guess execution \\
BRP & BR_PRED & Predictable branch of guess execution \\
CDE & CLK_DE_EN & PLE request is programmed \\
\hline
\end{tabular}

TABle 4: Partial Spark configuration parameters.

\begin{tabular}{lcc}
\hline Parameter name & Defaults & Description \\
\hline spark.driver.cores & 1 & Number of cores used for the driver process only in cluster mode \\
spark.driver.memory & $1 \mathrm{~g}$ & The amount of memory used for the driver process \\
spark.executor.memory & $1 \mathrm{~g}$ & The amount of memory used for the executor process \\
spark.executor.cores & All & Number of cores per executor \\
spark.reducer.maxSizeInFlight & $48 \mathrm{~m}$ & The maximum size of the map output obtained from each reduce task at the same time \\
spark.shuffle.compress & True & Whether to compress the mapping output file
\end{tabular}

and instruction characteristics. The characterization of these events reflects the performance characteristics of the Spark program running on the mobile processor. It is helpful to analyze the execution characteristics of big data tasks on mobile devices and improve the execution efficiency of big data tasks on mobile devices.

As shown in Table 3, it is introduced that the MEE module of MobilePerfMiner uses the XGBoost algorithm to establish a performance model for microarchitecture events and performance and obtains the importance ranking of the event characteristics of performance. In the process of feature extraction, according to the change of the error curve, we found three data features and explained the relationship between these three data features and the program and algorithm.

As shown in Table 4, Spark has been established as an attractive platform for big data analysis because it can hide most of the complexities related to parallelism, fault tolerance, and cluster settings from developers. However, this comes at the cost of having more than 150 configurable parameters, as shown in Table 4, which are some of the Spark configuration parameters. Due to the number of combinations of these parameters, it is not possible to fully examine the impact of these parameters. The default setting is to allow developers to quickly deploy their applications, but there is still the question of whether performance can be improved. 


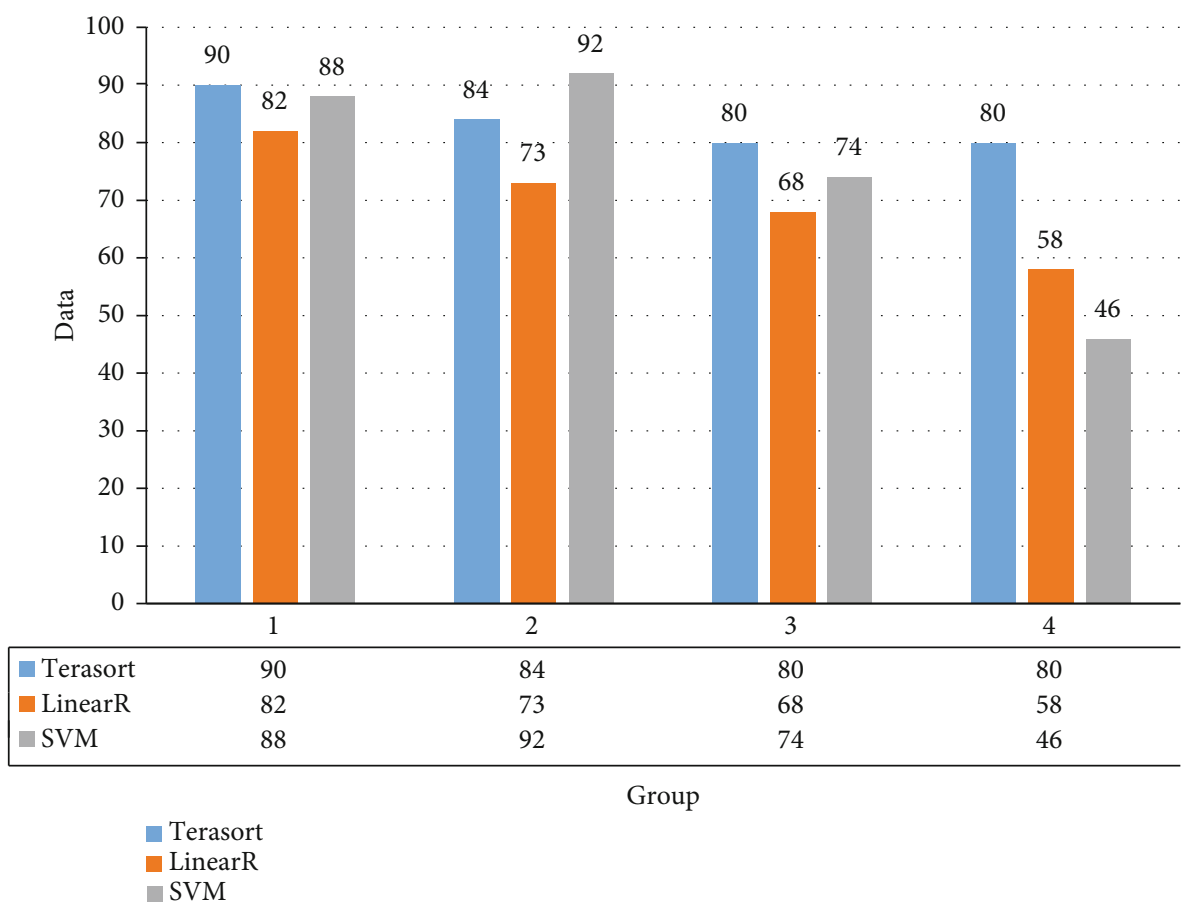

FIGURE 6: Runtime percentage diagram from different execution number parameters.

From the instruction characteristics of the Spark Bench program in Figure 6, we can see that branch instructions and floating-point instructions are key events in many programs. The number of CPU cores (spark.executor.cores) attribute of each executor controls the number of concurrent tasks that the executor can run. -spark.executor.cores 4 means that each executor can run up to 4 tasks at the same time. The number of cores affects the total number of instructions that the program can execute. The ARM Cortex-A9 mobile processor contains 4 processing cores (ARM Corporation, 2016). The picture shows the relationship between the running time ratio of Terasort, Linear Regression, and SVM programs with significant instruction characteristics and the number of execution cores. It can be seen that as the number of execution cores increases, the running time of the program decreases. When the number of execution cores increases to 4 , the execution time of Linear Regression and SVM programs is reduced by $50 \%$ compared with the number of execution cores, indicating branching and floating for Spark programs with point instruction features; the configuration parameter execution core number can improve program execution efficiency. The use of tuned configuration parameters saves an average of $36 \%$ of program execution time.

As shown in Figure 7, we can see the memory characteristics of the Spark program. On the one hand, the data memory barrier instruction is the performance bottleneck of the program. On the other hand, the on-chip multilevel cache system hinders memory access. Therefore, we appropriately adjust the proportion of storage and execution to memory and allocate memory reasonably. Shown in Figure 7 is a comparison of program execution time obtained with different memory configurations. For strongly connected component and Terasort programs, the program execution time gradually increases from 0.3 , while for SVDPlusPlus, the program execution time gradually decreases, showing that tuning the memory ratio can improve the efficiency of program execution. For example, for strongly connected component program, the optimal memory ratio can save $54 \%$ of program execution time. After tuning the parameters, an average of $37 \%$ of the program execution time was saved.

This article proposes a spark program parameter tuning method based on event sequencing based on microarchitecture events. Generally speaking, due to Spark's RDD data structure, data shuffling behavior, compression, and serialization parameters have a greater impact on performance. The serialization method has the greatest impact on CPU performance. Take Terasort as an example. The important events in the Terasort program are IFR (INS_FP_RR, the number of floating-point instructions through the register renaming phase) and ITA (INS_TLB_ALLOC, the number of TLB applications for instruction requests), indicating that the floating-point instructions of the program are an important event that affects performance, and the program optimizes the dependency of floating-point instructions during execution, so that it can be executed more efficiently in the out-of-order pipeline, so the structure of the optimized program can be Better reduce the blockage of the assembly line. The number of applications for TLB indicates that there are many instructions in the program that affect the performance of the virtual address translation physical address. Therefore, the capacity of the TLB table entry and the locality of page table access can be used to optimize the locality of the program. To reduce the number of TLB applications.

From Figure 8, we can see that the results of the above optimization we have reached the conclusion that the 


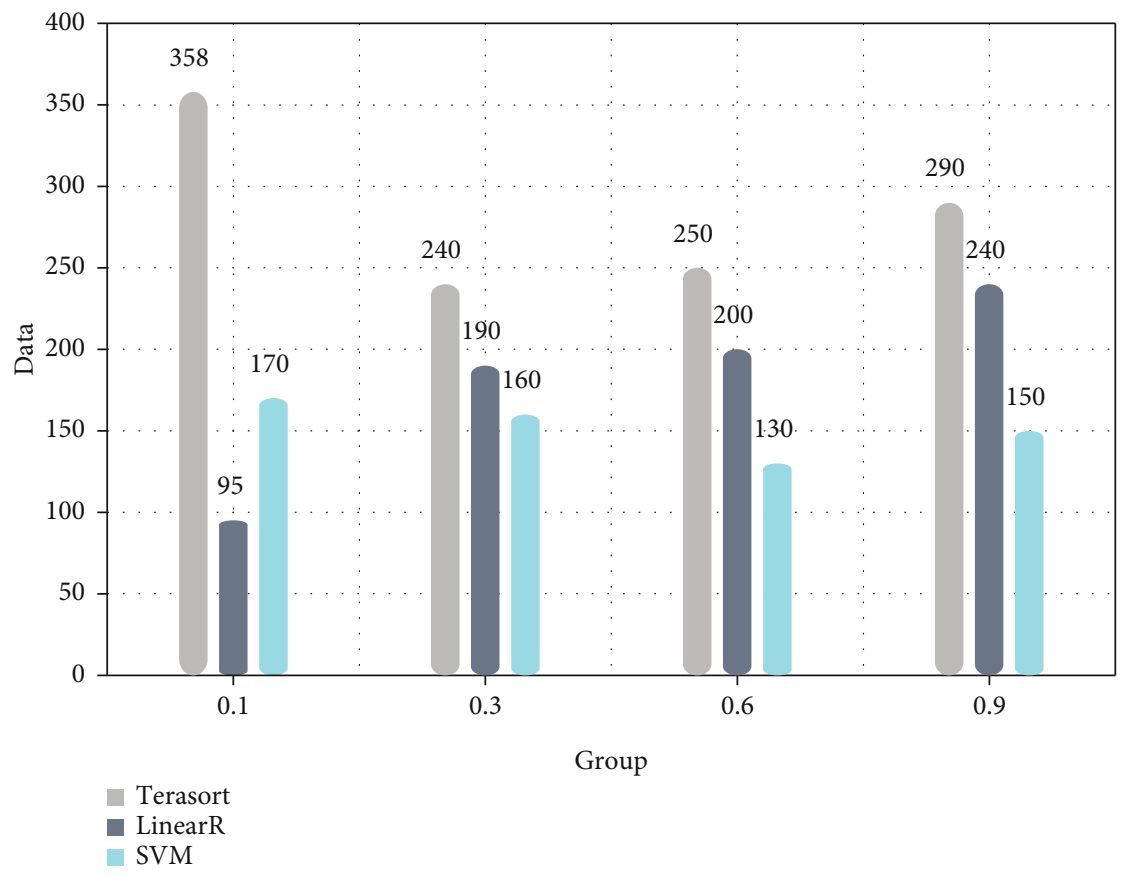

FIgURE 7: Runtime percentage diagram from different rate of memory.

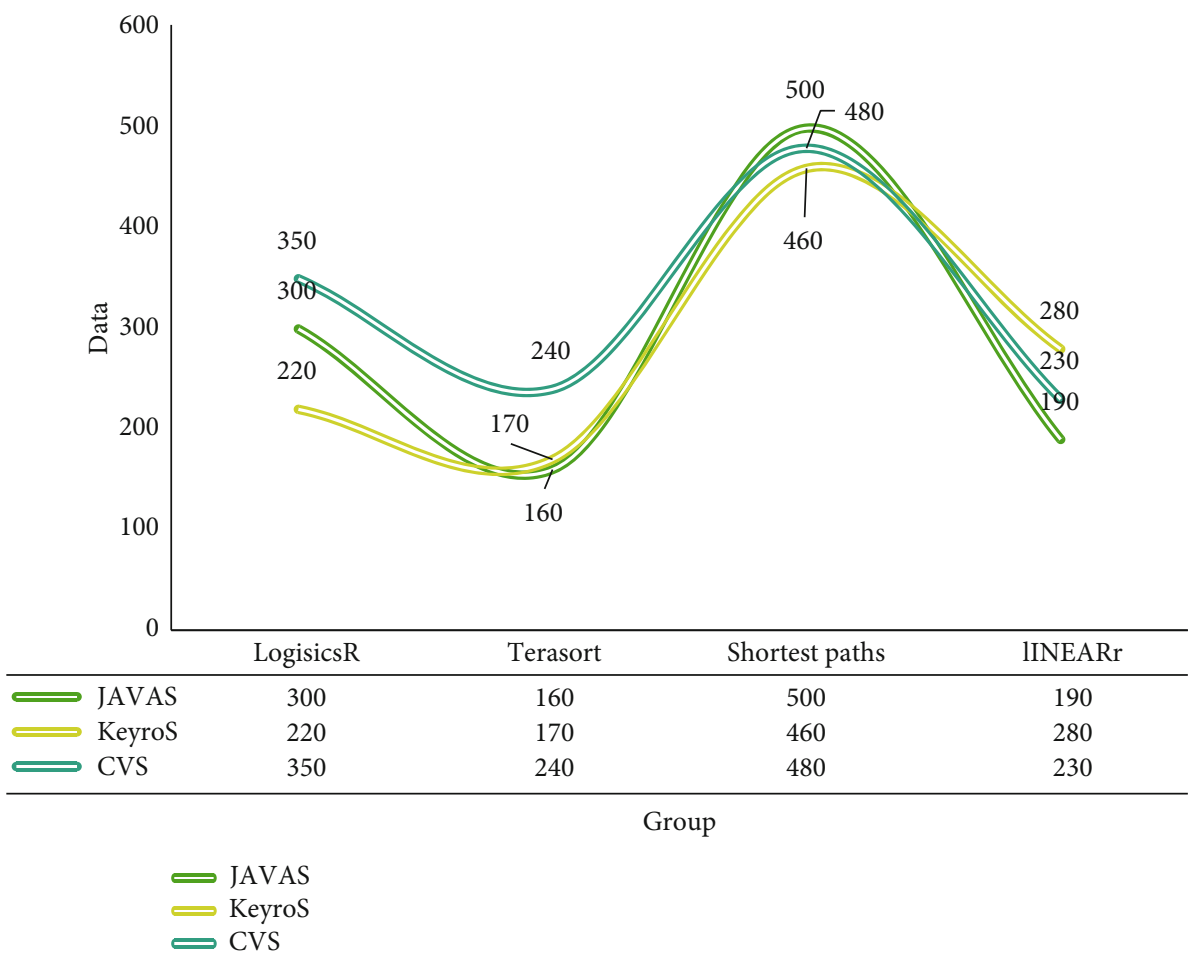

Figure 8: The optimization effect of serialization parameters is not obvious when the data set is small.

performance characteristics obtained by executing the program monitoring event and the configuration parameters correspond to the performance bottleneck. When the content of the table shows which event is the most important event, tuning the corresponding Spark parameters can reduce the execution time of the program and achieve the objective function shown in the formula. Executor core parameter can reduce the program execution time. How to use the performance characteristics obtained through MobilePerfMiner to tune Spark programs was introduced. According to the program performance characteristics obtained in Chapter 4, we start to optimize Spark configuration parameters from two aspects: instruction characteristics and memory system characteristics. For programs whose instruction feature is a 
key event, we optimized the configuration parameters of the number of execution cores, saving $36 \%$ of the average execution time. For programs with memory system characteristics, we optimized the memory weight configuration parameters, saving $37 \%$ of the program execution time on average. In addition, the data serialization parameter tuning has saved $21 \%$ of the program execution time.

\section{Conclusions}

This research investigated the characteristics and applications of ARM processor performance monitoring unit, hardware counter, and microarchitecture events and found that when running spark big data tasks on mobile platforms, hardware counters were used to measure events and performance characterization from the perspective of microarchitecture, of inadequacy. In order to understand the club's comprehensive ability, guide the club to develop its strengths and avoid weaknesses, and manage the athletes' athletic ability; this paper uses data mining technology to build a mining model to analyze the club's offensive and defensive balance ability, offensive combination ability, defensive ability, and psychological quality. This article firstly deals with the collected club technical statistics data and builds a mining model with the club's win or loss as the target attribute and the score as a decision attribute and analyzes the relationship between scores and wins. In order to study the comprehensive ability of the athletes and guide the club to manage the athletes' athletic ability more effectively, this article takes the core athletes of the club as the research object, uses data mining technology to construct a mining model, and analyzes the key ability and stability ability of the athletes. The disadvantage of this article is that the data studied are based on the original data obtained from the existing technical statistics database of the Tennis League. These data items are relatively fixed and cannot fully reflect the overall technical capabilities of the club or athletes; at the same time, some of the data is subject to the subjective judgment of the statistician. Impact, there are situations that are not objective enough, and the above problems will lead to incomplete or incomplete analysis results. Therefore, tennis coaches should strengthen the special physical fitness training of tennis players, improve the training plan, and learn from provinces and cities with higher levels of tennis, absorb advanced and excellent concepts, and improve the overall competitive level of national tennis. I will continue to work hard in the future, hoping to make my own contribution to the national tennis industry.

\section{Data Availability}

No data were used to support this study.

\section{Conflicts of Interest}

None of the authors have any conflicts of interest.

\section{Acknowledgments}

This work was supported by Xiangyang Science \& Technology Plan (high-tech field, Grant No. 2020ABH001191), Hubei University of Arts and Science Scientific Research Starting Foundation (Grant Nos. 2059073 and 2059074), the Basic Soft Science Research Projects of Wenzhou Science and Technology Bureau (Grant No. R20180012), the Zhejiang Philosophy and Social Sciences Foundation (Grant No. 19NDJC145YB), and Zhejiang Public Welfare Technology Application Research Project (Grant No. LGG19F020004).

\section{References}

[1] Y. Chen, W. Zheng, W. Li, and Y. Huang, "Large group activity security risk assessment and risk early warning based on random forest algorithm," Pattern Recognition Letters, vol. 144, pp. 1-5, 2021.

[2] S. Yang, "Volleyball player physical fitness evaluation model based on data mining," Modern Electronic Technology, vol. 40, no. 11, pp. 119-122, 2017.

[3] X. Yulin, "Research on the construction of special physical fitness indexes for professional tennis players," Diet Science, vol. 416, no. 24, p. 258, 2018.

[4] Z. Yang and Z. Yan, "Diagnosis and assessment of physical function status of excellent tennis players in the Graduate School of Wuhan Institute of Physical Education," West China Leather, vol. 40, no. 8, pp. 89-90, 2018.

[5] C. Zhuoheng, "Analysis of the principles and methods of physical fitness test based on association rules mining," China and Foreign Exchange, no. 30, pp. 30-31, 2017.

[6] Y. Hu, "The development and significance analysis of physical training in tennis training," Times Agricultural Machinery, vol. 45, no. 11, pp. 118-118, 2018.

[7] K. Yong and P. Jianjun, "Research on the evaluation index system of specialized physical fitness training for excellent tennis players in my country," Journal of Wuhan Institute of Physical Education, vol. 52, no. 11, pp. 88-94, 2018.

[8] X. Ao, L. Baiyan, and F. Wang, "Analysis of acupoint application in the treatment of tennis elbow based on modern literature," Jilin Journal of Traditional Chinese Medicine, vol. 38, no. 1, pp. 1-5, 2018.

[9] H. Fei, "Research on the optimization of the physical training of canoeists: taking the youth rowing team of the Chongming District Sports School in Shanghai as an example," Sports Fashion, vol. 2, pp. 33-34, 2020.

[10] Z. Luo, "Thinking of functional training on physical training of tennis players in colleges and universities- - taking the shoulder joint as an example," Stationery \& Sports Supplies \& Technology, vol. 13, no. 13, pp. 247-248, 2019.

[11] Z. Shuhua, "Design of college students' score data mining and physical fitness analysis system based on ID3 algorithm," Modern Electronic Technology, vol. 42, no. 5, pp. 104-106, 2019.

[12] L. Yichen, "Research on the body shape index of tennis players in Xi'an Institute of Physical Education," Sports Fashion, no. 3, pp. 210-210, 2018.

[13] Z. Dongbing, "Research on the evaluation model of volleyball mobilization training based on data mining," Computer Knowledge and Technology, vol. 16, no. 4, pp. 280-281, 2020. 
[14] L. Zhongqing, "Research on the disability mutual aid insurance compensation of my country's elite athletes based on data mining," Financial Theory and Practice, no. 11, pp. 96-99, 2017.

[15] H. Zhengying, "Research on the characteristics of tennis players' body shape and physical function," Sports Fashion, no. 4 , pp. 142-142, 2020.

[16] H. Wang, "Evaluation method of athlete's physical training effect based on Markov model," Journal of Chifeng University (Natural Science Edition), vol. 36, no. 3, pp. 96-99, 2020.

[17] X. Yani, "The effect of functional training on the physical fitness of college tennis students," Stationery, Sports \& Technology, vol. 444, no. 11, pp. 228-229, 2020.

[18] B. Liao, "Research on the characteristics of tennis players' body shape and physical function," Journal of Jiamusi Education College, no. 12, pp. 228-229, 2019.

[19] H. Rui and J. Yu, "Visual analysis of the research on "Tennis physical training" in my country," Sports Fashion, no. 11, pp. 68-68, 2019.

[20] B. Liao, "Research on the characteristics of tennis players' body shape and physical function," Journal of Jiamusi Vocational College, no. 12, pp. 219-220, 2019.

[21] Y. Qi, "Strategies and practice of optimizing physical fitness of elite athletes," China Sports Coaches, vol. 27, no. 1, pp. 21-25, 2019.

[22] Q. Jiaojiao, "Construction and simulation of athlete's multiattribute training data mining model based on improved Apriori algorithm," Microcomputer Applications, vol. 34, no. 12, pp. 140-142, 2018.

[23] W. Mofei, "Application of physical training in tennis training in colleges and universities," Science \& Technology Information, vol. 16, no. 22, pp. 226-227, 2018.

[24] C. Chen, "The impact of innovation in training methods on the improvement of tennis players' competitive ability-— taking the national soft net team as an example," Journal of Gansu Lianhe University (Natural Science Edition), vol. 32, no. 2, pp. 107-110, 2018.

[25] H. Luo, "Research on arc circle technology based on data mining algorithm," Journal of Fuyang Vocational and Technical College, vol. 29, no. 3, pp. 51-54, 2018.

[26] P. Yang, "Development and design based on the physical fitness test database system for table tennis players," Automation and Instrumentation, no. 1, pp. 50-51, 2017.

[27] Z. Shuyang, "Research on the training characteristics of tennis events," Tomorrow Fashion, no. 10, pp. 293-293, 2017.

[28] J. Yu and C. Jianyi, "Training and enlightenment of American junior tennis players," Journal of Shenyang Institute of Physical Education, vol. 36, no. 4, pp. 121-127, 2017.

[29] L. Lei and F. Yanhui, "Discussion on physical training of young tennis players," Journal of Hebei University of Engineering (Social Science Edition), vol. 34, no. 2, pp. 111-112, 2017.

[30] J. Cheng, "Research on the guiding ideology of physical training for young tennis players in my country," Exam Weekly, no. 24, pp. 139-139, 2018. 